\title{
THE HYPEROCTANT PROPERTY IN ORTHOMODULAR AC-LATTICES ${ }^{1}$
}

\author{
RONALD P. MORASH
}

\begin{abstract}
The complete atomic orthomodular lattice $L$ is said to have the hyperoctant property if and only if, for every orthogonal family of atoms $\left\{a_{\alpha}\right\}$ in $L$ with cardinality $>2$, there exists an atom $q$ such that $q<\bigvee_{\alpha} a_{\alpha}$ and $q \notin a_{\alpha}$ for each $\alpha$. The projection lattice of any separable Hilbert space has the hyperoctant property. In this paper, we show that an abstract complete atomic orthomodular lattice possessing the additional properties, $M$-symmetry, irreducibility, countably infinite dimension, and the angle bisection property, has the hyperoctant property. Additional remarks are made about the non- $M$-symmetric case.
\end{abstract}

1. Introduction. In his paper [4], D. E. Catlin defines, in a complete atomic orthomodular lattice $L$, the hyperoctant property (HP) by the condition: for any orthogonal family of atoms $\left\{a_{\alpha}\right\}$ in $L$ with cardinality $\geqslant 2$, there exists an atom $q$ such that $q \leqslant \bigvee_{\alpha} a_{\alpha}$ and $q$ fails to commute with any of the $a_{\alpha}$. This condition is clearly satisfied by the projection lattices of separable real, complex, and quaternionic Hilbert space. Also, it is worth mentioning that any complete atomic orthomodular lattice possessing HP also has the property that every interval in it is irreducible (cf. [3, Theorem 7] and [4, p. 414], " $\sigma-\mathrm{HP} \Rightarrow \mathrm{ABP} ")$. Catlin states as an open question, in essence (see [4, p. 414], "Does FHP $\Rightarrow \sigma$-HP?") whether an arbitrary complete atomic orthomodular lattice (containing a countably infinite orthogonal family of atoms, but no orthogonal family of atoms of cardinality $>\kappa_{0}$ ), in which every interval is irreducible, possesses HP. In our earlier paper [9], we posed the more restrictive question whether HP obtains in any infinite dimensional Hilbert lattice, that is, a lattice which is $M$-symmetric, in addition to having the other five properties just listed. (In the language of Maeda [8], $L$ is then a complete irreducible orthomodular AC-lattice.) This question was asked in connection with our conjecture, formulated in [9], that the three projection lattices are completely determined by the six lattice-theoretic properties. In this paper, we prove that the answer to the question on HP posed in [9] is "yes" under the further assumption of the angle bisection property (cf. [10], [11]). Since the only three known lattices having the first six properties also have the angle bisection property, and since the latter property is purely lattice-theoretic in

Received by the editors February 7, 1975.

AMS (MOS) subject classifications (1970). Primary 06A30; Secondary 46C05.

Key words and phrases. Orthomodular lattice, Hilbert lattice, coordinatization, hyperoctant property.

${ }^{1}$ This research was supported by a grant from the H. H. Rackham School of Graduate Studies of the University of Michigan.

C American Mathematical Society 1976 
nature, we are perfectly willing to assume it. Our derivation of HP uses coordinatization and a construction suggested by ideas of Amemiya and Araki [1]. A similar construction was employed by S. S. Holland, Jr. in his paper [5] to derive a result related to a possible classification theorem for Baer *-rings, similar to the one we have conjectured for orthomodular lattices.

2. The main theorem. Our principal result is this:

2.1. TheOREM. An infinite dimensional Hilbert lattice satisfying the angle bisection property possesses the hyperoctant property.

Assume $L$ is an infinite dimensional Hilbert lattice satisfying the angle bisection property. By [8, 34.5], there exists, corresponding to $L,\left(\left(k,{ }^{*}\right), V\right.$, $(),), k$ a division ring with involution $*, V$ a left vector space over $k$, such that $L$ is orthoisomorphic to the lattice $L_{\perp}\left(\left(k,{ }^{*}\right), V,(),\right)$ of all $\perp$-closed subspaces of $V, \perp$ being the orthogonality relation induced by the conjugate-bilinear, Hermitian (with respect to ${ }^{*}$ ) form $\left(\right.$, ) on $V$. Let $\left\langle a_{\alpha}^{\prime}\right\rangle$ represent an infinite orthogonal family of atoms in $L$. Since $L$ is separable, the family is necessarily countable. For each $n=1,2, \ldots$, let $e_{n}$ be a vector in $V$ such that $a_{n}^{\prime}=k e_{n}$, the one dimensional subspace of $V$ spanned by $e_{n}$. By the angle bisection property [11, p. 45], we may choose each $e_{n}$ so that $\left(e_{n}\right.$, $\left.e_{n}\right)=1$. Also, by [11, Theorem 3.1(a)], $k$ has characteristic zero and hence contains the rationals as a subfield (this is also a consequence of the angle bisection property).

Define two sequences of vectors $\left\langle a_{n}: n=1,2, \ldots\right\rangle$ and $\left\langle b_{m}: m=1\right.$, $2, \ldots>$ in $V$ by

$$
a_{n}=\sum\left(2^{1-k} e_{k}: k=1, \ldots, 2 n-1\right)+(1 / 3) 2^{3-2 n} e_{2 n}
$$

and

$$
b_{m}=(-1 / 3) e_{1}+\sum\left(2^{1-k} e_{k}: k=2, \ldots, 2 m\right)+(1 / 3) 2^{2-2 m} e_{2 m+1} .
$$

An easy calculation shows that $a_{n} \perp b_{m}$ for all $m, n=1,2, \ldots$ Note also that each vector is a finite linear combination of the $e_{n}$ and all coefficients are rational. Now let $A=\left\{b_{1}, b_{2}, \ldots\right\}^{\perp}$ and $B=A^{\perp}=\left\{b_{1}, b_{2}, \ldots\right\}^{\perp \perp}$ (which we note is clearly a subset of $\left.\left\{e_{1}, e_{2}, \ldots\right\}^{\perp \perp}\right)$. Clearly, $A$ and $B$ are orthogonal, both are $\perp$-closed subspaces of $V, a_{i} \in A$ and $b_{j} \in B$ for all $i$, $j=1,2, \ldots$ Since $L$ is assumed orthomodular, there must, in particular, exist vectors $a \in A, b \in B$ such that $e_{1}=a-b$. Let $q=k b$, so $q$ is an atom in $L$. We will show that $q$ satisfies the requirements of HP, with respect to the family $\left\langle a_{n}^{\prime}: n=1,2, \ldots\right\rangle$ of atoms initially chosen. We make the following evident observations about the vectors $a$ and $b$, and the atom $q$.

2.2. LEMMA. (i) $a-\left(a, e_{1}\right) e_{1}=b-\left(b, e_{1}\right) e_{1}$.

(ii) $\left(a, e_{j}\right)=\left(b, e_{j}\right)$ for all $j>1$.

(iii) For all $n=1,2, \ldots$, 


$$
a-\sum\left(\left(a, e_{k}\right) e_{k}: k=1, \ldots, n\right)=b-\sum\left(\left(b, e_{k}\right) e_{k}: k=1, \ldots, n\right) .
$$

(iv) $\left(a, e_{1}\right) \neq 0$ and $\left(b, e_{1}\right) \neq 0$.

(v) $q \leqslant V\left(a_{n}^{\prime}: n=1,2, \ldots\right)$.

Our goal now is to prove $q \not a_{n}^{\prime}$ for all $n=1,2, \ldots$ A crucial step in the proof is the following lemma, which gives information about the possible values of the coefficients $\left(a, e_{n}\right)$ and $\left(b, e_{n}\right)$. Since both $\left(b, e_{1}\right)$ and $\left(a, e_{1}\right)$ are nonzero, we may assume without loss of generality, that $\left(b, e_{1}\right)=-\frac{1}{3}$ and $(a$, $\left.e_{1}\right)=1+\varepsilon$, where $\varepsilon$ is an arbitrary element of $k$. We might point out that, in the actual Hilbert space case, if $a$ and $b$ are the vectors corresponding to the situation described here, $\left(b, e_{1}\right)=-\frac{1}{3},\left(a, e_{1}\right)=1$, and $\left(a, e_{n}\right)=\left(b, e_{n}\right)$ $=2^{1-n}$ for all $n \geqslant 2$, these vectors existing by Fourier expansions. Using the facts that $b \perp a_{i}$ for all $i=1,2, \ldots$ and $a \perp b_{j}$ for all $j=1,2, \ldots$, we will derive an expression in the variable $\varepsilon$ for each $\left(b, e_{i}\right), i \geqslant 2$, and thus for each $\left(a, e_{i}\right)$.

2.3. LemMA. For each $n=2,3,4, \ldots,\left(a, e_{n}\right)=\left(b, e_{n}\right)=2^{1-n}+2^{3-n} c_{n} \varepsilon$, where $c_{2}=0$ and $c_{n}=\frac{1}{5}\left(1-(-4)^{n-2}\right)$ for $n=3,4, \ldots$.

Proof. First, it is easy to verify directly that $\left(a, e_{2}\right)=\left(b, e_{2}\right)=\frac{1}{2}$, since the vectors $b$ and $a_{1}$ are orthogonal. Now suppose $n$ is even, $n>2$, and write $n=2 k$. Suppose inductively that $\left(a, e_{n-1}\right)=\left(b, e_{n-1}\right)=2^{2-n}+2^{4-n} c_{n-1} \varepsilon$, where $c_{n-1}=\frac{1}{5}\left(1-(-4)^{n-3}\right)$. Now, consider

$$
\begin{aligned}
0= & -0=\left(b, a_{k}\right)-\left(a, b_{k-1}\right) \\
= & \left(b, \sum\left(2^{1-i} e_{i}: i=1,2, \ldots, 2 k-1\right)+\frac{1}{3}\left(2^{3-2 k}\right) e_{2 k}\right) \\
& -\left(a,-\frac{1}{3} e_{1}+\sum\left(2^{1-i} e_{i}: i=2, \ldots, 2 k-2\right)+\frac{1}{3}\left(2^{4-2 k}\right) e_{2 k-1}\right) \\
= & \left(b, e_{1}\right)+\frac{1}{3}\left(a, e_{1}\right)+\left(2^{2-2 k}\left(b, e_{2 k-1}\right)-\frac{1}{3}\left(2^{4-2 k}\right)\left(a, e_{2 k-1}\right)\right) \\
& +\frac{1}{3}\left(2^{3-2 k}\right)\left(b, e_{2 k}\right) \\
= & -\frac{1}{3}+\frac{1}{3}(1+\varepsilon)+\left[\left(2^{2-n}-\frac{1}{3}\left(2^{4-n}\right)\right)\left(2^{2-n}+2^{4-n} c_{n-1} \varepsilon\right)\right] \\
& +\frac{1}{3}\left(2^{3-n}\right)\left(b, e_{n}\right) \\
= & \frac{1}{3} \varepsilon+\left[-\frac{1}{3}\left(2^{2-n}\right)\left(2^{2-n}+\frac{1}{5}\left(2^{4-n}\right)\left(1-(-4)^{n-3}\right) \varepsilon\right)\right] \\
& +\frac{1}{3}\left(2^{3-n}\right)\left(b, e_{n}\right) \\
= & \frac{1}{3} \varepsilon-\frac{1}{3}\left(4^{2-n}\right)-\frac{1}{15}\left(4^{3-n}\right)\left(1-(-4)^{n-3}\right) \varepsilon \\
& +\frac{1}{3}\left(2^{3-n}\right)\left(b, e_{n}\right) .
\end{aligned}
$$

Thus 


$$
\begin{aligned}
\left(b, e_{n}\right) & =3 \cdot 2^{n-3}\left(\frac{1}{3}\left(4^{2-n}\right)+\varepsilon\left(\frac{1}{15}\left(4^{3-n}\right)\left(1-(-4)^{n-3}\right)-\frac{1}{3}\right)\right) \\
& =2^{1-n}+3 \cdot 2^{n-3} \varepsilon\left(\frac{1}{15}\left(4^{3-n}\right)\left(\left(1-(-4)^{n-3}\right)-5\left(4^{n-3}\right)\right)\right) \\
& =2^{1-n}+\varepsilon\left(\frac{1}{5}\left(2^{3-n}\right)\left(\left(1-(-4)^{n-3}\right)-5.4^{n-3}\right)\right) \\
& =2^{1-n}+\varepsilon\left(\frac{1}{5}\left(2^{3-n}\right)\left(1-(-4)^{n-2}\right)\right) \\
& =2^{1-n}+2^{3-n} c_{n} \varepsilon,
\end{aligned}
$$

as desired. Note that the second last equality follows from the standard formula for the sum of a geometric series. If $n$ is odd, write $n=2 k+1$, and consider $0=\left(a, b_{k}\right)-\left(b, a_{k}\right)$. The result, in this case, follows much as before.

Note, for example, that $\left(a, e_{4}\right)\left(b, e_{4}\right)=\frac{1}{8}-\frac{3}{2} \varepsilon,\left(a, e_{5}\right)=\left(b, e_{5}\right)=\frac{1}{16}+\frac{13}{4} \varepsilon$, etc. From Lemma 2.3, it is clear that there is no value of $\varepsilon$ in $k$ such that $(b$, $\left.e_{n}\right)=0$ for all but a single value of $n$, hence we cannot have that the atom $q=a_{n}^{\prime}$ for any value of $n$. To prove $q \not a_{n}^{\prime}$ for all $n=1,2, \ldots$, it will therefore suffice to show that $q$ is not orthogonal to $a_{n}^{\prime}$, for each $n=1$, $2, \ldots$ Thus, we wish to consider whether a value of $\varepsilon$ is possible which would result in $\left(b, e_{n}\right)=0$ for any $n$ at all. Our claim is that no such $\varepsilon$ can actually occur. We will show that such an $\varepsilon$ forces $a$ and $b$ to fail to be orthogonal to each other-a contradiction since $a \in A, b \in B$, and $A \perp B$. Denote, for each $n=2,3,4, \ldots$, by $\varepsilon_{n}$ the element of $k$ such that $\left(b, e_{n}\right)=0$. Clearly, $\varepsilon_{n}=-\frac{1}{4} c_{n}^{-1}=-\frac{5}{4}\left(1-(-4)^{n-2}\right)^{-1}$. Note that each $\varepsilon_{n}$ is rational, and thus selfadjoint and central in $k$. Now denote, for each $n=3,4, \ldots$, by $f_{n}(\varepsilon)$ the inner product

$$
\left(\sum\left(\left(a, e_{i}\right) e_{i}: i=1,2, \ldots, n\right), \sum\left(\left(b, e_{i}\right) e_{i}: i=1,2, \ldots, n\right)\right) .
$$

Since the scalars $\left(a, e_{i}\right)$ and $\left(b, e_{i}\right)$ depend on $\varepsilon$, so does $f_{n}(\varepsilon)$, clearly. Also, if $\varepsilon$ is selfadjoint and central, so are the $\left(a, e_{i}\right)$ and $\left(b, e_{i}\right)$. In that event, $f_{n}(\varepsilon)$ equals

$$
-\frac{1}{3}(1+\varepsilon)+\frac{1}{4}+\left(\frac{1}{4}+\varepsilon\right)^{2}+\left(\frac{1}{8}-\frac{3}{2} \varepsilon\right)^{2}+\cdots+\left(2^{1-n}+2^{3-n} c_{n} \varepsilon\right)^{2},
$$

a quadratic in $\varepsilon$ of the form $A_{n} \varepsilon^{2}+B_{n} \varepsilon+C_{n}$, where each $A_{n}, B_{n}, C_{n}$ is rational. My claim is that, if for some $n>2, b$ were orthogonal to $e_{n}$, which would necessarily mean $\varepsilon=\varepsilon_{n}$, then $f_{n-1}\left(\varepsilon_{n}\right)$ would equal zero. But this would mean that

$$
\begin{aligned}
0=(a, b)=\left(a-\sum\left(\left(a, e_{i}\right) e_{i}: i=1,2, \ldots, n\right),\right. & \\
b & \left.-\sum\left(\left(b, e_{i}\right) e_{i}: i=1, \ldots, n\right)\right) .
\end{aligned}
$$

By Lemma 2.2(iii), this is a contradiction since (, ) has no isotropic vectors. Thus we need now only verify our claim that $f_{n-1}\left(\varepsilon_{n}\right)=0$ for $n \geqslant 3$. We do this by means of the slightly more general

2.4. LeMMA. The quadratic equation with rational coefficients $f_{n}(\varepsilon)=0$ has rational roots $\varepsilon_{n}$ and $\varepsilon_{n+1}$, for $n \geqslant 3$.

Proof. (i) Let $n=3$. Note that 


$$
\begin{aligned}
f_{3}(\varepsilon) & =-\frac{1}{3}(1+\varepsilon)+\frac{1}{4}+\left(\frac{1}{4}+\varepsilon\right)^{2}=\varepsilon^{2}+\frac{1}{6} \varepsilon-\frac{1}{48} \\
& =\left(\varepsilon+\frac{1}{4}\right)\left(\varepsilon-\frac{1}{12}\right)=0 \\
& \Leftrightarrow \varepsilon=-\frac{1}{4}=\varepsilon_{3} \quad \text { or } \varepsilon=\frac{1}{12}=\varepsilon_{4} .
\end{aligned}
$$

(ii) Using induction, suppose that $f_{n}(\varepsilon)$ has roots $\varepsilon_{n}$ and $\varepsilon_{n+1}$. Now $f_{n+1}(\varepsilon)$ $=f_{n}(\varepsilon)+\left(b, e_{n+1}\right)^{2}$. Since $\varepsilon_{n+1}$ is the root of $\left(b, e_{n+1}\right)^{2}=0$, then $\varepsilon_{n+1}$ is a root of $f_{n+1}(\varepsilon)=0$. We claim that $\varepsilon_{n+2}$ is the other root. Denoting $f_{n}(\varepsilon)$ by $A_{n} \varepsilon^{2}+B_{n} \varepsilon+C_{n}$, we note that

$$
C_{n}=-\frac{1}{3}+\frac{1}{3}\left(1-\left(\frac{1}{4}\right)^{n-1}\right)=-\left(\frac{1}{3}\right)\left(\frac{1}{4}\right)^{n-1},
$$

because each $C_{n}$ is simply the $n$th partial sum of the geometric series $-(1 / 3)+(1 / 2)^{2}+(1 / 4)^{2}+\ldots$ with first term $-1 / 3$ and ratio $1 / 4$. Since the product $\varepsilon_{n} \varepsilon_{n+1}$ of the roots of $f_{n}(\varepsilon)=0$ equals $C_{n} / A_{n}$, we must have that

$$
A_{n}=C_{n} / \varepsilon_{n} \varepsilon_{n+1}=-(1 / 75)\left(4^{3-n}\right)\left((-4)^{n-2}-1\right)\left((-4)^{n-1}-1\right) .
$$

Now

$$
\begin{aligned}
f_{n+1}(\varepsilon) & =f_{n}(\varepsilon)+\left(b, e_{n+1}\right)^{2} \\
& =A_{n}\left(\varepsilon-\varepsilon_{n}\right)\left(\varepsilon-\varepsilon_{n+1}\right)+\left(2^{-n}+2^{2-n} c_{n+1} \varepsilon\right)^{2} \\
& =A_{n}\left(\varepsilon-\varepsilon_{n}\right)\left(\varepsilon-\varepsilon_{n+1}\right)+4^{2-n} c_{n+1}^{2}\left(\varepsilon-\varepsilon_{n+1}\right)^{2} \\
& =\left(\varepsilon-\varepsilon_{n+1}\right)\left[A_{n}\left(\varepsilon-\varepsilon_{n}\right)+4^{2-n} c_{n+1}^{2}\left(\varepsilon-\varepsilon_{n+1}\right)\right]
\end{aligned}
$$

To complete our proof, we need only show that $\varepsilon_{n+2}$ is the root of the linear (in $\varepsilon$ ) equation $A_{n}\left(\varepsilon-\varepsilon_{n}\right)+4^{2-n} c_{n+1}^{2}\left(\varepsilon-\varepsilon_{n+1}\right)=0$. Now, by direct computation, $\varepsilon=\left(A_{n} \varepsilon_{n}+4^{2-n} c_{n+1}^{2} \varepsilon_{n+1}\right) /\left(A_{n}+4^{2-n} c_{n+1}^{2}\right)$. Substituting previously derived formulas, we find that $A_{n} \varepsilon_{n}=\left(1-(-4)^{n-1}\right) /\left(15 \cdot 4^{n-2}\right)$ and $4^{2-n} c_{n+1}^{2} \varepsilon_{n+1}=\left((-4)^{n-1}-1\right) /\left(5 \cdot 4^{n-1}\right)$ so that

$$
A_{n} \varepsilon_{n}+4^{2-n} c_{n+1} \varepsilon_{n+1}=\left(1-(-4)^{n-1}\right) /\left(15 \cdot 4^{n-1}\right) \text {. }
$$

Also $4^{2-n} c_{n+1}^{2}=(1 / 5)\left(4^{2-n}\left(1-(-4)^{n-1}\right)^{2}\right)$, so that

$$
\begin{aligned}
A_{n}+4^{2-n} c_{n+1}^{2}=(1 / 75)\left(4^{2-n}\right)[ & 3\left(1-(-4)^{n-1}\right)^{2} \\
& \left.-4\left((-4)^{n-2}-1\right)\left((-4)^{n-1}-1\right)\right] .
\end{aligned}
$$

Thus,

$$
\begin{aligned}
\varepsilon & =\frac{\left(1-(-4)^{n-1}\right)\left(75 \cdot 4^{n-2}\right)}{\left(15 \cdot 4^{n-1}\right)\left(\left[1-(-4)^{n-1}\right]\left[3\left(1-(-4)^{n-1}\right)+4\left((-4)^{n-2}-1\right)\right]\right)} \\
& =\frac{5}{4\left[3\left(1-(-4)^{n-1}\right)+4\left((-4)^{n-2}-1\right)\right]} \\
& =\frac{5}{4\left[-1-3(-4)^{n-1}+4(-4)^{n-2}\right]} \\
& =\frac{5}{4\left[-1-3(-4)^{n-1}-(-4)^{n-1}\right]}=\frac{5}{4\left(-1-4(-4)^{n-1}\right)}
\end{aligned}
$$




$$
=\frac{5}{\left((-4)^{n}-1\right) 4}=\frac{-5}{4\left(1-(-4)^{n}\right)}=\varepsilon_{n+2},
$$

as desired. The proof of Lemma 2.4 is complete. By the remarks preceding Lemma 2.4, Theorem 2.1 is also proved.

3. Concluding remarks. It is still not known to this writer whether Theorem 2.1 can be proved without the assumption of $M$-symmetry. The latter property certainly is not a necessary condition, in the presence of the other five properties, for HP. Consider, for instance, the horizontal sum of two copies of the projection lattice of complex, separable Hilbert space. This lattice possesses the other five properties of a Hilbert lattice, as well as HP, but is not $M$-symmetric (cf. [6, p. 416]). On the other hand, if the condition "every interval in $L$ is irreducible" is replaced by merely " $L$ is irreducible" (in the non- $M$-symmetric case), then HP need not obtain. Let $L$ be the horizontal sum of the power set of a countably infinite set with $2^{2}$. Then $L$ is a complete atomic irreducible orthomodular lattice. Furthermore $L$ contains a countably infinite orthogonal family of atoms, but no family of cardinality greater than $\kappa_{0}$. But clearly $L$ does not possess HP, nor is every interval in $L$ irreducible. The author is indebted to the referee for this example. We close by mentioning a problem related to the general Hilbert lattice classification problem. Our attempts to solve this problem led to the discovery of Theorem 2.1. Suppose that the infinite dimensional Hilbert lattice $L$ satisfying the angle bisection axiom is coordinatized, as before, by $\left(\left(k,{ }^{*}\right), V,(),\right)$. Suppose that $k$ is commutative and ${ }^{*}$ is the identity mapping. By [11, Theorem 3.1(a)], $k$ is formally real and so, by a theorem of Artin and Schreier [2, Satz 7b], admits an ordering. We ask whether the ordering is necessarily Archimedean. If so, then by a result of Holland [5, p. 519], we can conclude that $k$ is the real number field. If we assume that $k$ is commutative, ${ }^{*}$ is the identity mapping, and the ordering is non-Archimedean, we can prove, relative to the construction of $\$ 2$ of this paper, that $\varepsilon$ is an infinitesimal or is zero. Thus, for instance, the space of all "square-summable" sequences from $k, k$ a non-Archimedean ordered field, does not give rise to an orthomodular lattice. Other results, related to constructions from nonstandard analysis, which tend to suggest further that the answer to our question is "yes", are contained in my paper [12].

\section{REFERENCES}

1. I. Amemiya and H. Araki, A remark on Piron's paper, Publ. Res. Inst. Math. Sci. Ser. A 2 (1966/67), 423-427. MR 35 \#4130.

2. E. Artin and O. Schreier, Algebraische Konstruktion reeler Korper, Abh. Math. Sem. Univ. Hamburg 5 (1927), 85-99.

3. D. E. Catlin, Irreducibility conditions on orthomodular lattices, J. Nat. Sci. Math. 8 (1968), 81-87. MR 38 \# 2064.

4. _ Cyclic atoms in orthomodular lattices, Proc. Amer. Math. Soc. 30 (1971), 412-418. MR 44 \#2675.

5. S. S. Holland, Jr., Remarks on type I Baer and Baer *-rings, J. Algebra 27 (1973), 516-522. MR 48 \#8554.

6. M. D. MacLaren, Nearly modular orthocomplemented lattices, Trans. Amer. Math. Soc. 114 (1965), 401-416. MR 33 \#80. 
7. M. J. Maczynski, On a lattice theoretic characterization of Hilbert spaces, Colloq. Math. 31 (1974), 1-31.

8. F. Maeda and S. Maeda, Theory of symmetric lattices, Die Grundlehren der math. Wissenschaften, Band 173, Springer-Verlag, Berlin and New York, 1970. MR 44 \#123.

9. R. P. Morash, Orthomodularity and the direct sum of division subrings of the quaternions, Proc. Amer. Math. Soc. 36 (1972), 63-68. MR 47 \# 787.

10. , Angle bisection and orthoautomorphisms in Hilbert lattices, Canad. J. Math. 25 (1973), 261-272. MR 47 \#1698.

11. , Remarks on the classification problem for infinite-dimensional Hilbert lattices, Proc. Amer. Math. Soc. 43 (1974), 42-46.

12. _ Orthomodularity and non-standard constructions, Glasnik Mat. 10 (1975), 231-240.

Department of Mathematics and Statistics, University of Michigan, Dearborn, Michigan 48128 